\title{
Effective population sizes and adaptive genetic variation in a captive bird population
}

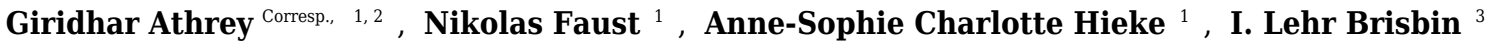 \\ ${ }^{1}$ Department of Poultry Science, Texas A\&M University, College Station, Texas, United States of America \\ 2 Faculty of Ecology and Evolutionary Biology, Texas A\&M University, College Station, Texas, USA \\ 3 Savannah River Ecology Lab, Aiken, South Carolina, United States \\ Corresponding Author: Giridhar Athrey \\ Email address: giri.athrey@tamu.edu
}

Captive populations are considered a key component of ex situ conservation programs. Research on multiple taxa have shown the differential success of maintaining demographic versus genetic stability and viability in captive populations. In typical captive populations, usually founded by few or related individuals, genetic diversity can be lost and inbreeding can accumulate rapidly, calling into question their ultimate utility for release into the wild. Furthermore, domestication selection for survival in captive conditions is another concern. Therefore, it is crucial to understand the dynamics of population sizes, particularly the effective population size, and genetic diversity at non-neutral and adaptive loci in captive populations.

In this study, we assessed effective population sizes and genetic variation at both neutral microsatellite markers, as well as SNP variants from the MHC-B locus of a captive Red Junglefowl population. This population is represents a rare instance of a population with a well-documented history in captivity, following a realistic scenario of chain-of-custody, unlike many captive lab populations. Our analyses, which included 27 individuals comprising the entirety of one captive population show very low neutral and adaptive genetic variation, as well as low effective sizes, which correspond with the known demographic history. Finally, our study also shows the divergent impacts of small effective size and inbreeding in captive populations on microsatellite versus adaptive genetic variation in the MHC-B locus. Our study provides insights into the difficulties of maintaining adaptive genetic variation in small captive populations. 
1 Effective population sizes and adaptive genetic variation in a captive bird population

2

3 Giridhar Athrey ${ }^{1,2+}$, Nikolas Faust ${ }^{1}$, Anne-Sophie Charlotte Hieke ${ }^{1}$, I. Lehr Brisbin ${ }^{3}$

$4 \quad{ }^{1}$ Department of Poultry Science, Texas A\&M University, College Station, TX.

$5 \quad{ }^{2}$ Faculty of Ecology and Evolutionary Biology, Texas A\&M University, College Station, TX.

$6 \quad{ }^{3}$ Savannah River Ecology Lab, Aiken, SC.

7

$8{ }^{+}$Corresponding author: Giridhar Athrey, Department of Poultry Science, Texas A\&M

9 University, 2472 TAMU, College Station, TX. Email: giri.athrey@tamu.edu

10

11

12

13

14

15

16

17 


\section{Abstract}

19 Captive populations are considered a key component of ex situ conservation programs. Research 20 on multiple taxa have shown the differential success of maintaining demographic versus genetic 21 stability and viability in captive populations. In typical captive populations, usually founded by

22 few or related individuals, genetic diversity can be lost and inbreeding can accumulate rapidly, 23 calling into question their ultimate utility for release into the wild. Furthermore, domestication 24 selection for survival in captive conditions is another concern. Therefore, it is crucial to 25 understand the dynamics of population sizes, particularly the effective population size, and 26 genetic diversity at non-neutral and adaptive loci in captive populations.

27 In this study, we assessed effective population sizes and genetic variation at both neutral 28 microsatellite markers, as well as SNP variants from the MHC-B locus of a captive Red 29 Junglefowl population. This population is represents a rare instance of a population with a well30 documented history in captivity, following a realistic scenario of chain-of-custody, unlike many 31 captive lab populations. Our analyses, which included 27 individuals comprising the entirety of 32 one captive population show very low neutral and adaptive genetic variation, as well as low 33 effective sizes, which correspond with the known demographic history. Finally, our study also 34 shows the divergent impacts of small effective size and inbreeding in captive populations on 35 microsatellite versus adaptive genetic variation in the MHC-B locus. Our study provides insights into the difficulties of maintaining adaptive genetic variation in small captive populations. 


\section{Introduction}

39

40

41

42

43

44

45

46

47

48

49

50

51

52

53

54

\section{Genetics of captive populations}

The management of demographic security and genetic diversity are among the central considerations in conservation (Ralls \& Ballou, 1986). Captive populations have long played a key role in conservation as a management tool to ensure demographic security (Hedrick 1992). The earliest work in conservation of endangered species focused on breeding in captivity to increase population sizes and for eventual release back into the wild. However demographic security and the maintenance of genetic diversity can sometimes be at odds, as genetic diversity is derived from the effective population size $\left(\mathrm{N}_{\mathrm{e}}\right)$. One issue in captive populations is the differential reproductive success of some breeding pairs that are better suited to captive conditions, leading to selection for survival in captive environments. This phenomenon can further reduce the $\mathrm{N}_{\mathrm{e}}$ due to founder effects (Nei, Maruyama \& Chakraborty, 1975; Newman \& Pilson, 1997). From the perspective of the reintroduction of the offspring of such captive breeders back into the wild, this may be a less than ideal situation, as these captive-adapted offspring may not carry the adaptive genetic variation required for survival in their natural habitat. Historically, captive individuals were raised for the genetic support of threatened wild populations, and to maintain genetic compatibility (prevent outbreeding depression) and genetic variation (ability to adapt to natural environment). However, these objectives can be materialized only if genetic diversity can be sustained, and if genetic drift and inbreeding can be limited. The relationship between population bottlenecks and reduction in effective population sizes and genetic diversity has been reported from various vertebrate taxa (Leberg, 1992; Athrey et al., 2011, 2012; Lovatt \& Hoelzel, 2014). The loss of genetic diversity due to drift and inbreeding in captive populations has also been well documented (Willoughby et al., 2015, 2017) and the 
61 drawbacks of using closed captive breeding populations for reintroduction have been described

62 (Lynch \& O'Hely, 2001). These range from the rapid decline of fitness upon reintroduction

63 (Araki, Cooper \& Blouin, 2007) to a range of consequences for genetic diversity (Witzenberger

64 \& Hochkirch, 2011). Witzenberger et al. (2011) also suggest the need for assessment of genetic

65 diversity before ex situ conservation programs. The consensus of these studies suggest that

66 considerations of genetic diversity of captive populations may be as crucial as maintaining

67 demographic stability, but is perhaps much more challenging than the maintenance of

68 demographic stability, because mating among relatives is not entirely avoidable. While some

69 studies have focused on assessing genetic diversity, in many instances the history of the founding

70 individuals is not well known, or the studbook data may be incomplete (Witzenberger \&

71 Hochkirch, 2011). Additionally, inbreeding estimates may assume that founders are unrelated

72 (Ruiz-López et al., 2009). For example, Alcaide et al. (2010) compared wild versus captive and

73 reintroduced Kestrel, but in that instance, it is not clear how many generations the kestrels had

74 bred in captivity. In another case study using experimental captive breeding, Willoughby et al.

75 (2017) report on white-footed mice populations that were bred in captivity for 20 generations,

76 and were able to observe mitigating effects on genetic diversity using a very deliberate mating

77 protocol (mean-kinship method). However, in many instances, there may be no choice available

78 as to the source of individuals obtained and used in captive breeding programs. Furthermore, in

79 extreme cases, small numbers of breeding individuals may have to be maintained in captivity for

80 decades until suitable habitat and molecular/technological tools to enable ex situ applications

81 become applicable. From that perspective, it is essential to have realistic estimates of how much

82 genetic diversity is maintained, and whether estimates of genetic diversity at neutral markers are

83 informative about variation at loci crucial for survival in the wild. 
84

85 In this study, we used a captive population of the Red Junglefowl (Gallus gallus murghi) to characterize the impacts of un-managed captive breeding over a period of 57 years (38-57 generations). The study population called the Richardson Strain (RRJF) has a well-documented history since its import to the United States in 1961. The history of this population is illustrated as a flowchart in Figure 1. We investigated the consequences of captive breeding on genetic diversity at neutral and adaptive genetic markers experienced by this population since the early 91 1960s.

Demographic history of Richardson's Red Junglefowl (RRJF)

94 The study population of Red Junglefowl was initially imported from Northern India in 1961 as 95 part of the U.S. Fish and Wildlife's Foreign Game Introduction Program (FGIP) and documented in USFWS Special Reports (Bump \& Bohl, 1964). More recently, the history of the population in

97 the United States was documented in detail in the graduate thesis of Tomas Condon (Condon, 98 2012). Briefly, the birds that were imported to the United States were captured in the Indian states of Bihar and Uttar Pradesh (now Uttarakhand), between 1959-1961. A total of 119 survived the capture and shipping to the United States. These birds were placed in hatcheries across nine different states, and over the next decade, approximately 10,000 birds were released into U.S. habitats for hunting, until the FGIP program ended. Over the next four decades, almost all the descendant RJF a) failed to survive in their new habitats, b) became incorporated into

104 private aviaries, or c) remained in small breeding populations maintained by dedicated 105 researchers and aviculturists. All the surviving RJF of known ancestry (estimated around 100106 150) were derived from a small number of birds. These birds have a well documented chain of 
107 custody. In 1969, I. Lehr Brisbin (of the Savannah River Ecology Lab) received five birds from

108 the South Carolina Department of Wildlife Resources, to which 26 were added in 1970. By 1971,

109 this flock numbered only eight adult birds (four of each sex). While more hatchlings were added

110 to this in mid-1971, the population remained at under 15 individuals, until they were transferred

111 to Mr. Isaac Richardson of Alabama. Mr. Richardson started his colony with 12 individuals (8

112 males). Between 1972-2010, the colony was sustained by Mr. Richardson, which later became

113 known as the Richardson's Red Junglefowl (RRJF). Over this time, the population expanded and

114 was distributed to a few others, but at any time, the breeding colony never exceeded 20

115 individuals (Condon, 2012). Based on behavioral and morphological studies of Red Junglefowl,

116 it has been argued that the RRJF represent a pure and unique population of Red Junglefowl

117 (Brisbin \& Peterson, 2007), whose preservation is critical in the face of hybridization with

118 domestic chickens in the native range of Junglefowl (Peterson \& Brisbin, 1998; Brisbin \&

119 Peterson, 2007).

120

121 The research colony maintained at Texas A\&M University (TAMU) is one of the two known

122 remaining populations that trace their ancestry back to the survivors of Mr. Richardson's flock.

123 The TAMU colony was established with two males and three females and expanded to 27

124 individuals at the time of the study. All available individuals were included in this present study.

125

126 Genetic diversity and effective population sizes

127 As the management of genetic diversity is one of the primary intentions of captive breeding

128 programs, it is important to assess the consequences of captive breeding over multiple

129 generations on genetic diversity. Other authors have shown reductions in genetic diversity in 
130 captive populations, and these typically result from differential survival and increased genetic

131 drift, but also a form of (unintentional) domestication selection (Briscoe et al., 1992;

132 Montgomery et al., 2010). In these instances, it is important to characterize what the effective

133 population size is, as this one parameter can drive various other genetic parameter estimates. The

134 effective population size $\left(N_{e}\right)$ can be defined as the size of an ideal population that experiences

135 the same amount of genetic drift or inbreeding as the actual (census) population (Wright, 1932,

136 1948). While a number of approaches have been developed to estimate $\mathrm{N}_{\mathrm{e}}$ - for example,

137 temporal estimates based on two or more samples, methods based on linkage disequilibrium, and

138 coalescent approaches (Wang, Santiago \& Caballero, 2016). Due to our access to a single point

139 in time, as well as our interest in understanding recent effective population size, we focused only

140 on methods for contemporary or recent $\mathrm{N}_{\mathrm{e}}$ estimates. In this study, we evaluated genetic

141 diversity, estimated the effective population size $\left(\mathrm{N}_{\mathrm{e}}\right)$, and analyzed linkage disequilibrium using

142 two types of markers - namely microsatellite markers and SNP markers. The SNP markers

143 assayed genetic diversity along chromosome 16 (details in methods), a microchromosome which

144 contains both the MHC class I and class II genes, in addition to olfactory receptors and

145 scavenger proteins (Miller \& Taylor, 2016). The MHC (major histocompatibility complex) is a

146 collection of genes that form a crucial part of vertebrate adaptive immunity, and in chicken they

147 have been demonstrated to be important in resistance against diseases such as Marek's Disease

148 (Shiina, Hosomichi \& Hanzawa, 2006; Miller \& Taylor, 2016).

\section{Materials and methods}

150 Study Population

151 A total of 27 birds bred in captivity at the Texas A\&M Poultry Research Center were genotyped

152 in this study. The population represents three generations. The parental cohort was comprised of 
1532 males and three females in January 2016 and had expanded to 27 individuals by the second

154 half of 2017. At the time of sampling, the population included original parents (used to establish

155 colony), in addition to two subsequent generations that were hatched in Texas. All individuals

156 available were included in this study.

157 Molecular Methods

158 Venal blood was sampled from all individuals by puncturing the brachial vein and stored in the

159 Tris-EDTA based stabilization solution, Longmire Buffer, until further processing (Longmire et

160 al., 1997). Birds were sampled in accordance with protocols approved by the Texas A\&M

161 Institutional Animal Care and Use Committee (IACUC 2016-0065). Genomic DNA was isolated

162 from whole blood using the DNeasy Blood and Tissue kit (Qiagen Inc). For microsatellite

163 genotyping, a total of 18 microsatellite markers were selected from a list of known chicken

164 microsatellite loci (Tadano et al., 2007) and screened for the presence of polymorphic loci. The

165 selection of loci was based on our intention to characterize marker variation across the genome.

166 The 18 loci screened were each from a different chromosome, and were picked based on their

167 reported polymorphism. Of the screened loci, three loci were monomorphic in our study

168 population, or were not amplified by PCR consistently and were not used further (full list and

169 primer sequences presented in S1). To generate genotype data from microsatellite loci, we used

170 the fluorescently labeled M13 method (Schuelke 2000), which allows greater flexibility of

171 fluorescent dye tagging for multiplexing. PCR thermoprofile was optimized based on published

$172 \mathrm{~T}_{\mathrm{m}}$ for each primer pair (and considering M13 sequence), and amplification was performed in a

$17325 \mu 1$ reaction using NEB Taq Polymerase with reaction buffer (New England Biolabs) and NEB

174 dNTPs (New England Biolabs). PCR amplification was performed on an Eppendorf

175 Mastercycler Pro thermal cycler (Eppendorf, Hauppauge, NY), running an initial denaturation at 
$17695^{\circ} \mathrm{c}$ for 5 minutes, followed by 35 cycles of denaturation $\left(95^{\circ} \mathrm{c}, 30 \mathrm{~s}\right)$, annealing $\left(\mathrm{T}_{\mathrm{m}}, 10 \mathrm{~s}\right)$, and

177 extension $\left(72^{\circ} \mathrm{c}, 45 \mathrm{~s}\right)$, and a final extension for 10 minutes.

178 Each microsatellite locus was amplified and then pooled for multiplex genotyping based on

179 combinations of fluorescently labeled probes. Multiplex genotyping was performed on the ABI

1803730 capillary analyzer at the DNA sequencing facility on Science Hill (Yale University, New

181 Haven, CT) for fragment analysis. For SNP analyses, equimolar DNA isolates were submitted to 182 the molecular genetics lab at Hy-Line International for genotyping using a custom SNP panel

183 (Fulton et al., 2016b; Nguyen-Phuc, Fulton \& Berres, 2016). The SNP panel was developed as

184 an assay for the Major Histocompatibility Complex - B locus (MHC-B), based on the

$185 \mathrm{KASP}^{\mathrm{TM}}$ chemistry (LGC group, UK) where each allele is identified by its fluorescent label

186 (VIC or FAM). A total of 90 known SNP loci are genotyped using this panel and covers the

187 region between basepairs 30,189 to 240,933 on chromosome 16 . While microsatellite loci came

188 from multiple chromosomes (Table S1), all the SNP loci are from a single gene-rich

189 microchromosome, and as is typical for SNP datasets, the markers represent a mix of

190 intronic/intergenic (putatively neutral) and coding regions.

191 Genetic Analyses

192 The raw data was downloaded into the software Geneious (Biomatters, New Zealand) and 193 analyzed using the microsatellite plugin. Allele bins, based on peak data from across all samples, 194 were created for each locus. Following this, every individual biological sample was processed 195 through the peak calling step. Once the automatic peak calls were obtained, every allele call was 196 manually verified to check for errors. The final allele calls were exported into the MS Excel197 based tool, GENALEX v6.5 (Peakall \& Smouse, 2006). The data were then exported to the 198 GENEPOP format for estimation of the effective population size. The SNP calls were generated 
199 (by Hy-Line International) using the Kraken software (LGC group, UK). IUPAC base calls

200 were first re-coded manually into 2-letter genotypes, and then imported into GENALEX and

201 recoded into a numeric format for further analysis.

202 Estimation of expected and unbiased expected heterozygosity $\left(\mathrm{H}_{\mathrm{e}}\right.$ and $\left.\mathrm{uH} \mathrm{H}_{\mathrm{e}}\right)$, and measures of the

203 inbreeding coefficient $\mathrm{F}_{\text {IS }}$ were both calculated within GENALEX, based on 999 permutations of

204 the data. These parameters were estimated for both the microsatellite and SNP data

205 independently.

206 Effective Population Size

207 Next, to estimate the effective population size of this captive-bred population, we used three 208 different estimators that are known to estimate contemporary or recent effective population sizes 209 - namely the heterozygosity excess (HE) method (Zhdanova \& Pudovkin, 2008; Pudovkin, 210 Zhdanova \& Hedgecock, 2010), the linkage disequilibrium (LD) method (Waples \& DO, 2008)

211 and the molecular coancestry (MC) method (Nomura, 2008). All three $\mathrm{N}_{\mathrm{e}}$ estimates were

212 generated using the software program NeEstimator, version 2 (Do et al., 2014). These

213 estimators are expected to estimate the population size based on shared alleles (MC) or the signal

214 of genetic drift - either due to allele frequency differences among parents (HE) or due to the

215 linkage disequilibrium among markers (LD). In this case, we also know which assumptions were

216 violated with some certainty. For example, this captive population has been closed to

217 immigration and not subject to artificial selection (except unintentional domestication selection

218 in captivity), but the assumption of random mating is likely to be violated, as we expect this

219 population to have an increased frequency of mating among relatives over the last five decades.

220 While it is known that most real natural populations may violate one or more assumptions of an

221 ideal population, the departure from random mating is expected to result in underestimates of $\mathrm{N}_{\mathrm{e}}$ 
222 (Waples, Antao \& Luikart, 2014). Similarly, the molecular coancestry estimate is expected to be

223 biased downward in inbred populations (Nomura, 2008). Considering the differential

224 consequences of these assumptions for our captive population, we estimated $\mathrm{N}_{\mathrm{e}}$ using the three

225 methods mentioned above. The analyses were carried out separately for the microsatellite data,

226 and for the SNP data. Furthermore, we were particularly interested in determining the $\mathrm{N}_{\mathrm{e}}$ as

227 obtained from markers from a single chromosome. By definition, a chromosome is a single

228 linkage group (Groenen et al., 2000; Wright et al., 2010), and linkage of loci over a single

229 chromosome can be expected to be stabler over evolutionary time, compared to markers sampled

230 from across the genome. However, estimates of contemporary $\mathrm{N}_{\mathrm{e}}$ are expected to be biased

231 downward by the length of the chromosome, and also the number of chromosomes (Waples,

232 Larson \& Waples, 2016) primarily due to physically linked loci, and recombination frequencies

233 being lower on short chromosomes. To our knowledge, this is the first study where a high

234 density of markers from a single chromosome was used to explore the population history. For

235 estimation of $\mathrm{N}_{\mathrm{e}}$, we used the phased genotypes from each individual as input (details below).

237 Haplotype and Linkage Analyses

238 To understand the effects of captive breeding on the number and structure of unique haplotypes

239 in the captive population, we first phased the SNP genotype data to identify the unique

240 haplotypes present in the population. We used the program PHASE v2 (Stephens, Smith \&

241 Donnelly, 2001) to phase the SNP loci into unique haplotype sequences. We performed phasing

242 and estimation of haplotype frequencies from five replicate runs of the program PHASE. Each

243 replicate run started with a different seed and was comprised of 1000 iterations, with a thinning

244 interval of five and a burn-in interval of 100. Following the completion of these runs, the number 
245 of unique haplotypes and their frequencies were recorded and checked across replicate runs. As

246 the number of haplotypes and their frequencies was very consistent across runs, we determined

247 that increasing the run length was not necessary (Stephens, Smith \& Donnelly, 2001). The list of

248 unique haplotypes was then used to construct a haplotype network using the 'APE' package on

249 the R statistical platform (Paradis, Claude \& Strimmer, 2004; Popescu, Huber \& Paradis, 2012).

250 This approach constructs a matrix of Kimura-2 parameter genetic distance (Kimura 1980) based

251 on nucleotide differences among the haplotypes and plots a network representing differences

252 among haplotypes. We also estimated nucleotide diversity $(\pi)$, haplotype diversity, and estimate

253 of Tajima's D, using the R package PEGAS (Paradis, 2010).

254

255 Finally, to examine linkage structure across chromosome 16 based on the 90 SNP loci, we used

256 the program HaploView (Barrett et al., 2005). This program summarizes estimated Minor Allele

257 Frequency (MAF) for each locus and generates haplotype and linkage information for locus

258 pairs, which are then represented in a graphical format. Higher values of linkage disequilibrium

259 (LD) suggest stronger association among loci, and in turn, indicate low recombination between

260 loci.

\section{Results}

262 Genetic Diversity

263 For the microsatellite data, $93.3 \%$ of the loci were polymorphic, and the three estimates of

264 heterozygosity based on microsatellite markers were very similar to each other (observed $=0.359$,

265 expected $=0.371$, unbiased $=0.379$ ). For the SNP dataset, only $37.8 \%$ of the loci were

266 polymorphic, which translated into heterozygosity estimates of $0.107,0.096$, and 0.098

267 (observed, estimated, and unbiased, respectively, Table 1). Estimates of F, the fixation 
268 index/inbreeding coefficient, were also divergent between the microsatellite (0.065) and SNP

269 datasets (-0.137). Positive values of $\mathrm{F}$ are typically indicative of more significant inbreeding than

270 expected, whereas negative values suggest more outbreeding than expected. This latter result is

271 potentially a consequence of the low observed and expected heterozygosities for the SNP dataset,

272 arising from the small proportion of SNP loci that were polymorphic.

273 Unique Haplotypes and Linkage

274 As expected, analysis of linkage disequilibrium at MHC-linked SNP loci on chromosome 16

275 revealed very high D' (linkage disequilibrium) values across all pairwise comparisons. D' was

276 estimated to have a value of 1 , indicating little or no recombination between marker pairs (Figure

277 2). Such high values indicate that as a consequence of captive breeding in a small founding

278 population, individuals in this population have inherited virtually identical chromosomes

279 (chromosome 16 at least), a phenomenon that would be indistinguishable from the absence of

280 recombination.

281 Analysis of haplotypes in the study population using the program PHASE revealed five unique

282 haplotypes (Figure 3), and of these only three were found at frequencies higher than $10 \%$, with

283 the most common haplotype represented in $70 \%$ of individuals (haplotype 1 ). The next two most

284 common haplotypes were found at approximately $14 \%$ frequency each. These haplotypes suggest

285 at best three unique haplotype lineages contributing to the population and presumably do not

286 reflect novel haplotypes emerged by mutations since the initial population contraction. Estimate

287 of nucleotide diversity $(\pi)$ was 0.009 , whereas the haplotype diversity was 0.43 . Finally, we

288 found a significantly negative Tajima's D based on the haplotype sequences $-3.19(\mathrm{P}<0.01)$,

289 suggesting a genetic bottleneck (or directional selection). Comparison of nucleotide diversity and

290 haplotypes at MHC loci from wild jungefowl and domestic chicken from other studies are shown 
291 in Table 2.

292

293 Effective Population Size estimates

294 The $\mathrm{N}_{\mathrm{e}}$ estimates were less than 10 for the microsatellite marker dataset, except for the

295 heterozygote excess method (Table 3). For the single chromosome SNP dataset, both the LD and

296 MC estimates were 1 or lower with narrow 95\% intervals, whereas the $\mathrm{H}_{\mathrm{E}}$ estimator was in the

297 low single digits $\left(\mathrm{N}_{\mathrm{eHE}}=4.8,95 \% \mathrm{CI}=3.1-11\right)$. If these estimates are compared against the total

298 number of individuals in the local population (Texas $A \& M$ ), the $N_{e} / N_{c}$ ratios range from about

$2990.14-0.27$ for microsatellite markers, whereas they range from $0.007-0.17$ for the SNP based

300 estimates.

301

302

303

304

305 Discussion

306 Our study generated valuable new understanding of genetic diversity and effective population

307 sizes in populations that have persisted in captivity for a fairly small number of generations

$308(<60)$. Additionally, our comparison of genome wide neutral microsatellite diversity, and high-

309 density SNP survey of adaptive genetic loci revealed important differences in how these marker

310 types respond to population bottlenecks, and how that is reflected in the estimates of effective

311 population size; while microsatellite-based genetic diversity estimates were low, they were, 
312 nonetheless, higher than SNP-based estimates for the MHC-linked region. These differences

313 were also reflected in the population histories revealed by these markers; effective size estimates

314 were extremely low for the MHC-linked SNP loci. The disparity in estimates between the neutral

315 and adaptive marker types is potentially concerning from the perspective of captive population

316 based ex situ conservation programs, and also has implications for endangered wild populations

317 with small numbers of breeding individuals. One important suggestion is that estimates of

318 functional genetic diversity and effective population sizes are overestimated by microsatellite 319 loci.

320

321 Genetic Diversity

322 We found relatively low genetic diversity based on heterozygosity, among both marker types,

323 but estimates of heterozygosity were higher using microsatellite markers compared to MHC-

324 linked SNP loci. This is not a surprising result, as microsatellites may not be highly correlated

325 with underlying genomic diversity (Väli et al., 2008), for example, due to ascertainment bias for

326 polymorphic loci. Tokarska et al. (2009) have also shown that in species or populations with low

327 genetic diversity, heterozygous SNP loci may be more informative about population history and

328 structure. In our case, the MHC-linked SNP marker panel included loci from both coding and

329 noncoding regions, but all these markers came from a single chromosome.

330 While it is difficult in our study to determine how the MHC-linked SNP loci may be

331 representative of whole genome diversity, in this study population we expect that chromosome

33216 would be experiencing selection or drift in ways that are consistent with the rest of the

333 genome. The MHC is a set of important adaptive loci (Flajnik \& Kasahara, 2001), and in natural

334 populations they may be expected to experience selection pressures distinct from the rest of the 
335 genome (Sutton et al., 2011; Strand et al., 2012; Oliver \& Piertney, 2012). In chicken, the MHC

336 loci have been shown to be important for adaptive immune responses against a number of

337 infectious diseases, and hence is a target of both natural and artificial selection (Kroemer et al.,

338 1990; Kaufman \& Wallny, 1996; Shiina et al., 2007; Miller \& Taylor, 2016). But, in this captive

339 population with a history of small population size, and high degree of nonrandom mating, we

340 expect drift to be a predominant evolutionary force. Furthermore, selection prior to a population

341 bottleneck is expected to result in a disproportionate loss of diversity at MHC loci (Sutton et al.,

342 2011). The recorded history does not show if any major selection events occurred immediately

343 prior to the FGIP importation, but the breeding and release programs between 1962-1970 do not

344 suggest any disease related mortality events (Bump \& Bohl, 1964; Condon, 2012). The

345 demographic history following arrival in the United States is suggestive mainly of random

346 genetic drift. Hence we believe that the measures of diversity based on the MHC-liked SNP

347 panel to be reflective of genome-wide patterns of diversity (both adaptive and neutral), while the

348 microsatellite-based estimates are likely to represent genome-wide neutral marker diversity

349 alone. If this supposition is correct, then assessing and, perhaps managing, genetic diversity at

350 loci that underpin adaptive traits would be as important as assessing neutral genetic diversity, if

351 not more so.

352 The second conclusion of the comparison between microsatellite markers and SNP markers is

353 the potential implications of such disparity for other endangered or threatened species of

354 conservation interest. Several studies have used microsatellite markers to assess genetic variation

355 following population contractions (Bouzat, Cheng \& Lewin, 1998; Wisely, Buskirk \& Fleming,

356 2002; Johnson \& Dunn, 2006; Athrey et al., 2011; Hammerly, Morrow \& Johnson, 2013). Due to

357 the relatively low cost and accessibility of microsatellite loci, the usage of these markers has 
358 become ubiquitous in population and conservation genetics, and in the assessment of genetic

359 diversity. If microsatellite-based measures of genetic diversity or effective population sizes are

360 higher than SNP-based genetic diversity, as observed in the current study, it would be potentially

361 concerning from the perspective of measuring the genetic viability of wild populations.

362

363 Effective Size estimates

364 We found low (single digit) $\mathrm{N}_{\mathrm{e}}$ estimates across all estimators. On the one hand this is consistent 365 with the known demographic history of few breeding individuals at any given time, on the other

366 hand, low estimates may arise from small sample size or other conditions. We expect $\mathrm{N}_{\mathrm{e}}$

367 estimates to be biased downward due to the small number of individuals in this study, but these

368 sample sizes are not unusual in endangered populations. Several of the $\mathrm{N}_{\mathrm{e}}$ estimators expect

369 biased estimates at sample sizes under $\mathrm{N}=50$. However, in this study, $100 \%$ of individuals were

370 sampled. Another source of bias, especially for the LD method, as reported by Waples et al.

371 (2016), is the number and length of chromosomes. In our case, the SNP data were generated

372 from chromosome 16 - one of the smaller micro-chromosomes, which has an estimated length of

373 48-80cM (Burt \& Cheng, 1998; Groenen et al., 2000). We expect both the smaller sample size

374 and the use of single chromosome SNP markers to be contributing to low $\mathrm{N}_{\mathrm{e}}$ estimates using the

375 LD, and perhaps the MC method.

376 Given the known history of the study population, the low $\mathrm{N}_{\mathrm{e}}$ estimates were not unexpected, but

377 the differences between the microsatellite markers from across the genome, and the single

378 chromosome SNP markers were notable, and reveal different population histories. Both the MC

379 and the LD methods estimated $\mathrm{N}_{\mathrm{e}}$ to be equal to or lower than one, based on the SNP dataset.

380 Whether these differences are the result of different recombination frequencies for marker types 
381 or actual biological lineages for unique haplotypes is challenging to differentiate. Our finding of

382 a low number of MHC haplotypes in this population suggests that fewer unique haplotypes to be

383 driving the observed pattern.

384 Due to our exact knowledge of the census size, the $\mathrm{N}_{\mathrm{e}} / \mathrm{N}_{\mathrm{c}}$ ratios are informative about both the 385 processes of marker inheritance, as well as the performance of the estimators themselves.

386 Secondly, all the three estimators used in this study are expected to represent the previous or 387 recent parental generations. Therefore, for both the LD and MC methods, we believe that the 388 estimates obtained here represent the founding event of the Texas population in 2016. On the 389 other hand, as changes in heterozygosity occur more gradually over time, and the accumulation 390 of inbreeding may take several generations, the HE estimator may be more representative of a

391 founder event bounded by the initial founding of the U.S. populations in 1961. However, as our 392 analyses of haplotypes and recombination frequencies showed, all the MHC-linked SNP loci are 393 in very high linkage disequilibrium. Such lack of independence among loci in the entire 394 population will be indistinguishable from an effective size of one, which is what we observe 395 here. Our results point to one of the limitations of LD methods in populations such as the study 396 population, where the founders represent only five unique haplotypes. Secondly, the MHC 397 region is inherited as haplotypes instead of segregating loci along the region (Hosomichi et al., 398 2008), which can also bias estimates of LDNe.

399 Finally, the number of microsatellite loci and the low diversity at these loci might be another 400 source of bias in the estimates. Even though we chose microsatellite loci that were reported to be 401 highly polymorphic, these loci had low diversity in the study population. While Antao et al. 402 (2011) have showed that sample size is more crucial for detection of population size declines 403 (especially with LD methods), in our case we sampled the entire census population size. While 
404 these methods are ideally suited for estimating $\mathrm{N}_{\mathrm{e}}$, when the true $\mathrm{N}_{\mathrm{e}}$ is low $(<100)$, real world

405 scenarios such as our study population (both the census and effective sizes are low) pose

406 challenges for the application and interpretation of $\mathrm{N}_{\mathrm{e}}$ estimates, and show the need for

407 development of methods to assess and correct for bias in such situations.

408

409 Haplotype diversity and linkage

410 We observed only three major haplotypes in the population, and it will be valuable to understand

411 how these haplotypes are maintained in subsequent generations. We believe that the low

412 haplotype diversity is a consequence of the history of captive breeding among a small number of

413 individuals, rather than a reflection of the source populations. Haplotype diversity in other wild

414 junglefowl populations has been reported to be quite high. For example, Nguyen-Phuc et al.

415 (2016) reported 313 unique haplotypes from 199 wild-caught individuals from Vietnam. This

416 suggests that the progenitors of the RRJF are likely to have come from a similarly diverse

417 population, which has since lost genetic diversity in captivity. It is also worth noting that the

418 genetic bottleneck and the resulting reduction in genetic diversity in only 50 years perhaps

419 mirrors the early genetic history during selection for domestication, except for periodic gene

420 flow from wild individuals. It would appear that such immigration was necessary to maintain the

421 high levels of genetic diversity that is found in domestic chickens today (Granevitze et al., 2007;

422 Fulton et al., 2016b).

423 We observed high linkage disequilibrium among loci in the SNP dataset - an estimate that is

424 known to be inflated when using small sample sizes, and very likely to be contributing to the

425 high D’ values observed (Ardlie, Kruglyak \& Seielstad, 2002; England et al., 2006). Taken

426 together with the reduced haplotype diversity in this population, however, the linkage 
427 disequilibrium estimates are biologically plausible.

428 Selection for tameness and success in captive environments is inevitable in captive breeding

429 populations (Frankham et al., 1986; Briscoe et al., 1992; Montgomery, DA Briscoe \& Frankham, 430 2002). The genetic consequences of domestication have received much attention in recent years

431 and the genetic architecture of traits - mainly driven by linkage and pleiotropy are considered to

432 be crucial in the expression of specific phenotypes in domesticated varieties. For example,

433 Wright et al. (2010) showed strong linkage blocks, as well as low heterozygosity regions

434 associated with selective sweeps in domesticated varieties of chicken. The haplotype diversity

435 and linkage patterns we observed in our captive population appear to be consistent with what

436 might occur early during domestication, if a small number of individuals are selected. The high

437 genetic diversity found in domestic chicken breeds and wild junglefowl populations, and also at

438 the MHC-linked SNP loci (Fulton et al., 2016a, 2017; Nguyen-Phuc, Fulton \& Berres, 2016)

439 would suggest that domestication of chicken would have included frequent immigration over

440 several tens of generations. One glimpse at this is found in the estimates of Tajima's D from

441 Wild Junglefowl (Nguyen-Phuc, Fulton \& Berres, 2016) versus the captive junglefowl

442 population (this study). Wild junglefowl populations show positive values of Tajima's D,

443 suggesting diversifying or balancing selection (Tajima, 1989), whereas both the domestic

444 chicken and captive junglefowl show negative Tajima's D values (Table 2). While we cannot

445 distinguish between the effects of directional selection and bottleneck in our study, the latter is

446 expected given the known history of this population. The major shift in diversity from wild to

447 captive populations within 60 generations is intriguing, especially in the context of domestication

448 selection. 


\section{Conclusions}

451 Our study showed the low genetic diversity and small effective population sizes in a captive

452 breeding bird population. Furthermore, our study was able to relate effective population sizes to 453 the known demographic history of the captive population. Given this history, the low effective 454 population size estimates we found are not surprising. Furthermore, the low genetic diversity, 455 and haplotype diversity both indicate that these populations are indeed pure, and unmixed with 456 domestic varieties, since their initial importation to the United States. Our study shows the rapid 457 loss of genetic diversity in captive populations in only a few generations (50-57 generations 458 since arrival importation), which can be particularly concerning from the standpoint of ex situ 459 conservation goals starting with captive populations. Finally, we showed how captive breeding 460 populations, even in the absence of any intentional selection, can affect linkage structure across a 461 set of adaptive genetic loci, and potentially reduce the fitness and adaptability of captive-born 462 individuals upon release back into the wild.

\section{Acknowledgements}

464 The authors thank Dr. Town Peterson, and Shawna Marie Hubert for comments on an earlier 465 draft of the manuscript. We also thank Dr. Janet Fulton of Hy-Line International for her support 466 of this project.

469 Table 1: Estimates of genetic diversity for both microsatellite and SNP datasets are presented.

470 For each marker type, genetic diversity measures are shown along with their standard errors. 
471 The columns present the number of alleles (Na), effective number of alleles (Nae), observed

472 heterozygosity (Ho), expected heterozygosity (He), the unbiased expected heterozygosity (uHe),

473 and the fixation index $F$.

474 Table 2: Estimates of nucleotide diversity, haplotype number and diversity and Tajima's $D$ in

475 captive study population, compared against wild junglefowl populations and domestic chicken.

476 All the studies compared here used the same high-density SNP panel covering the MHC-B locus

477 on Chromosome 16.

478 Table 3: Estimates of effective population size Ne are presented for microsatellite and SNP

479 datasets. Ne estimates from three estimators are shown along with their respective 95\%

480 confidence intervals. Ne was estimated using the Heterozygote Excess (NeHE), Linkage

481 Disequilibrium (NeLD), and the Molecular Coancestry (NeMC) methods.

482

483 Figure Legends

484 A graphical representation of the known population history of the Richardson's Red Junglefowl

485 population in captivity and chain of custody. The red rimmed box denotes released populations

486 that ultimately failed to take hold, and experienced extinction in their new habitat. The blue

487 shaded boxes show the history of the populations that trace back to Isaac Richardson's flock.

488

489 A linkage map based on the 90 SNP loci on chromosome 16, generated from genotype data for

490 the study population. The map shows pairwise estimates of linkage disequilibrium between 
491

492

493

494

495

496

497

498

499

500

501

502

503

504

505

506

507

508

509

510

511

512

513

514

515

516

517

518

519

520

521

522

markers. Red colored blocks suggest high linkage disequilibrium (D') values of 1, implying little to no recombination between markers.

A haplotype network of the the five (phased) haplotypes detected in the study population. Only three of the five unique haplotypes were found at a frequently of over $10 \%$ in the population (I, III, and V).

\section{Bibliography}

Alcaide, M., J. J. Negro, D. Serrano, J. L. Antolín, S. Casado, and M. Pomarol. 2010. Captive breeding and reintroduction of the lesser kestrel Falco naumanni: a genetic analysis using microsatellites. Conserv. Genet. 11:331-338.

Antao, T., A. Pérez-Figueroa, and G. Luikart. 2011. Early detection of population declines: high power of genetic monitoring using effective population size estimators. Evol. Appl. 4:144-154.

Araki, H., B. Cooper, and M. S. Blouin. 2007. Genetic effects of captive breeding cause a rapid, cumulative fitness decline in the wild. Science 318:100-103.

Ardlie, K. G., L. Kruglyak, and M. Seielstad. 2002. Patterns of linkage disequilibrium in the human genome. Nat. Rev. Genet. 3:299-309.

Athrey, G., K. R. Barr, R. F. Lance, and P. L. Leberg. 2012. Birds in space and time: genetic changes accompanying anthropogenic habitat fragmentation in the endangered black-capped vireo (Vireo atricapilla). Evol. Appl. 5:540-552.

Athrey, G., D. L. Lindsay, R. F. Lance, and P. L. Leberg. 2011. Crumbling diversity: comparison of historical archived and contemporary natural populations indicate reduced genetic diversity and increasing genetic differentiation in the golden-cheeked warbler. Conserv. Genet. 12:13451355.

Barrett, J. C., B. Fry, J. Maller, and M. J. Daly. 2005. Haploview: analysis and visualization of LD and haplotype maps. Bioinformatics 21:263-265.

Bouzat, J. L., H. H. Cheng, and H. A. Lewin. 1998. Genetic evaluation of a demographic bottleneck in the greater prairie chicken. Conservation ....

Brisbin, I. L., and A. T. Peterson. 2007. Playing chicken with red junglefowl: identifying phenotypic markers of genetic purity in Gallus gallus. Animal Conservation 10:429-435.

Briscoe, D. A., J. M. Malpica, A. Robertson, G. J. Smith, R. Frankham, R. G. Banks, and J. S. F. Barker. 1992. Rapid Loss of Genetic Variation in Large Captive Populations of Drosophila Flies: 
523 Implications for the Genetic Management of Captive Populations. Conservation Biology 6:416524425.

525 Bump, G., and W. H. Bohl. 1964. Summary of foreign game bird propagation andliberations

5261960 to 1963 . U.S. Fish \& Wildlife Service, Washington, D.C.

527 Burt, D. W., and H. H. Cheng. 1998. The chicken gene map. ILAR journal.

528 Condon, T. 2012. Morphological detection of genetic introgression in red junglefowl (gallus

529 gallus). Georgia Southern University.

530 Do, C., R. S. Waples, D. Peel, G. M. Macbeth, B. J. Tillett, and J. R. Ovenden. 2014.

531 NeEstimator v2: re-implementation of software for the estimation of contemporary effective

532 population size (Ne ) from genetic data. Mol. Ecol. Resour. 14:209-214.

533 England, P. R., J.-M. Cornuet, P. Berthier, D. A. Tallmon, and G. Luikart. 2006. Estimating

534 effective population size from linkage disequilibrium: severe bias in small samples. Conserv.

535 Genet. 7:303-308.

536 Flajnik, M. F., and M. Kasahara. 2001. Comparative genomics of the MHC: glimpses into the 537 evolution of the adaptive immune system. Immunity 15:351-362.

538 Frankham, R., H. Hemmer, O. A. Ryder, E. G. Cothran, M. E. Soulé, N. D. Murray, and M.

539 Snyder. 1986. Selection in captive populations. Zoo Biol. 5:127-138.

540 Fulton, J. E., M. E. Berres, J. Kantanen, and M. Honkatukia. 2017. MHC-B variability within the

541 Finnish Landrace chicken conservation program. Poult. Sci. 96:3026-3030.

542 Fulton, J. E., A. R. Lund, A. M. McCarron, K. N. Pinegar, D. R. Korver, H. L. Classen, S.

543 Aggrey, C. Utterbach, N. B. Anthony, and M. E. Berres. 2016a. MHC variability in heritage

544 breeds of chickens. Poult. Sci. 95:393-399.

545 Fulton, J. E., A. M. McCarron, A. R. Lund, K. N. Pinegar, A. Wolc, O. Chazara, B. Bed'Hom,

546 M. Berres, and M. M. Miller. 2016b. A high-density SNP panel reveals extensive diversity,

547 frequent recombination and multiple recombination hotspots within the chicken major

548 histocompatibility complex B region between BG2 and CD1A1. Genet. Sel. Evol. 48:1.

549 Granevitze, Z., J. Hillel, G. H. Chen, and N. T. K. Cuc. 2007. Genetic diversity within chicken 550 populations from different continents and management histories. Animal ....

551 Groenen, M. A., H. H. Cheng, N. Bumstead, B. F. Benkel, W. E. Briles, T. Burke, D. W. Burt, L.

552 B. Crittenden, J. Dodgson, J. Hillel, S. Lamont, A. P. de Leon, M. Soller, H. Takahashi, and A.

553 Vignal. 2000. A consensus linkage map of the chicken genome. Genome Res. 10:137-147.

554 Hammerly, S. C., M. E. Morrow, and J. A. Johnson. 2013. A comparison of pedigree- and DNA555 based measures for identifying inbreeding depression in the critically endangered Attwater's 556 Prairie-chicken. Mol. Ecol. 22:5313-5328.

557 Hedrick, P. W. 1992. Genetic conservation in captive populations and endangered species. Pp.

558 45-68 in S. K. Jain and L. W. Botsford, eds. Applied Population Biology. Springer Netherlands, 559 Dordrecht.

560 Hosomichi, K., M. M. Miller, R. M. Goto, Y. Wang, S. Suzuki, J. K. Kulski, M. Nishibori, H. 561 Inoko, K. Hanzawa, and T. Shiina. 2008. Contribution of mutation, recombination, and gene 562 conversion to chicken MHC-B haplotype diversity. J. Immunol. 181:3393-3399. 
563 Johnson, J. A., and P. O. Dunn. 2006. Low Genetic Variation in the Heath Hen Prior to

564 Extinction and Implications for the Conservation of Prairie-Chicken Populations. Conserv.

565 Genet. 7:37-48.

566 Kaufman, J., and H. J. Wallny. 1996. Chicken MHC molecules, disease resistance and the 567 evolutionary origin of birds. Pp. 129-141 in O. Vainio and B. A. Imhof, eds. Immunology and

568 developmental biology of the chicken. Springer Berlin Heidelberg, Berlin, Heidelberg.

569 Kimura, M. 1980. A simple method for estimating evolutionary rates of base substitutions

570 through comparative studies of nucleotide sequences. J. Mol. Evol. 16:111-120.

571 Kroemer, G., A. Bernot, G. Behar, A.-M. Chausse, L.-N. Gastinel, F. Guillemot, I. Park, P.

572 Thoraval, R. Zoorob, and C. Auffray. 1990. Molecular genetics of the chicken MHC: current

573 status and evolutionary aspects. Immunol. Rev. 113:119-145.

574 Leberg, P. L. 1992. Effects of population bottlenecks on genetic diversity as measured by

575 allozyme electrophoresis. Evolution.

576 Longmire, J. L., R. J. Baker, M. Maltbie, and Texas Tech University. 1997. Use of "Lysis

577 Buffer" in DNA isolation and its implication for museum collections /. Museum of Texas Tech

578 University, Lubbock, TX. :

579 Lovatt, F. M., and A. R. Hoelzel. 2014. Impact on Reindeer (Rangifer tarandus) Genetic

580 Diversity from Two Parallel Population Bottlenecks Founded from a Common Source. Evol.

581 Biol. 41:240-250.

582 Lynch, M., and M. O’Hely. 2001. Captive breeding and the genetic fitness of natural 583 populations. Conservation Genetics 2:363-378.

584 Miller, M. M., and R. L. Taylor. 2016. Brief review of the chicken Major Histocompatibility 585 Complex: the genes, their distribution on chromosome 16, and their contributions to disease 586 resistance. Poult. Sci. 95:375-392.

587 Montgomery, M. E., DA Briscoe, and R. Frankham. 2002. Rapid genetic deterioration in captive 588 populations: causes and conservation implications. Conservation ....

589 Montgomery, M. E., L. M. Woodworth, P. R. England, D. A. Briscoe, and R. Frankham. 2010. 590 Widespread selective sweeps affecting microsatellites in Drosophila populations adapting to 591 captivity: Implications for captive breeding programs. Biological Conservation 143:1842-1849.

592 Nei, M., T. Maruyama, and R. Chakraborty. 1975. The bottleneck effect and genetic variability 593 in populations. Evolution 29:1-10.

594 Newman, D., and D. Pilson. 1997. Increased probability of extinction due to decreased genetic 595 effective population size: experimental populations of clarkia pulchella. Evolution 51:354-362.

596 Nguyen-Phuc, H., J. E. Fulton, and M. E. Berres. 2016. Genetic variation of major

597 histocompatibility complex (MHC) in wild Red Junglefowl (Gallus gallus). Poult. Sci. 95:400598411.

599 Nomura, T. 2008. Estimation of effective number of breeders from molecular coancestry of 600 single cohort sample. Evol. Appl. 1:462-474.

601 Oliver, M. K., and S. B. Piertney. 2012. Selection maintains MHC diversity through a natural 602 population bottleneck. Mol. Biol. Evol. 29:1713-1720. 
603 Paradis, E. 2010. pegas: an R package for population genetics with an integrated-modular 604 approach. Bioinformatics 26:419-420.

605 Paradis, E., J. Claude, and K. Strimmer. 2004. APE: Analyses of Phylogenetics and Evolution in 606 R language. Bioinformatics 20:289-290.

607 Peakall, R. O. D., and P. E. Smouse. 2006. genalex 6: genetic analysis in Excel. Population 608 genetic software for teaching and research. Mol. Ecol. Notes 6:288-295.

609 Peterson, A. T., and I. L. Brisbin. 1998. Genetic endangerment of wild Red Junglefowl Gallus 610 gallus? Bird Conservation International.

611 Popescu, A.-A., K. T. Huber, and E. Paradis. 2012. ape 3.0: New tools for distance-based 612 phylogenetics and evolutionary analysis in R. Bioinformatics 28:1536-1537.

613 Pudovkin, A. I., O. L. Zhdanova, and D. Hedgecock. 2010. Sampling properties of the 614 heterozygote-excess estimator of the effective number of breeders. Conservation Genetics.

615 Ralls, K., and J. Ballou. 1986. Captive breeding programs for populations with a small number 616 of founders. Trends Ecol Evol (Amst) 1:19-22.

617 Ruiz-López, M. J., E. R. S. Roldán, G. Espeso, and M. Gomendio. 2009. Pedigrees and 618 microsatellites among endangered ungulates: what do they tell us? Mol. Ecol. 18:1352-1364.

619 Shiina, T., W. E. Briles, R. M. Goto, K. Hosomichi, K. Yanagiya, S. Shimizu, H. Inoko, and M. 620 M. Miller. 2007. Extended gene map reveals tripartite motif, C-type lectin, and Ig superfamily 621 type genes within a subregion of the chicken MHC-B affecting infectious disease. J. Immunol. 622 178:7162-7172.

623 Shiina, T., K. Hosomichi, and K. Hanzawa. 2006. Comparative genomics of the poultry major 624 histocompatibility complex. Animal Sci. J. 77:151-162.

625 Stephens, M., N. J. Smith, and P. Donnelly. 2001. A new statistical method for haplotype 626 reconstruction from population data. Am. J. Hum. Genet. 68:978-989.

627 Strand, T. M., G. Segelbacher, M. Quintela, L. Xiao, T. Axelsson, and J. Höglund. 2012. Can 628 balancing selection on MHC loci counteract genetic drift in small fragmented populations of 629 black grouse? Ecol. Evol. 2:341-353.

630 Sutton, J. T., S. Nakagawa, B. C. Robertson, and I. G. Jamieson. 2011. Disentangling the roles of 631 natural selection and genetic drift in shaping variation at MHC immunity genes. Mol. Ecol. 632 20:4408-4420.

633 Tadano, R., M. Nishibori, N. Nagasaka, and M. Tsudzuki. 2007. Assessing genetic diversity and 634 population structure for commercial chicken lines based on forty microsatellite analyses. Poult. 635 Sci. 86:2301-2308.

636 Tajima, F. 1989. Statistical method for testing the neutral mutation hypothesis by DNA 637 polymorphism. Genetics 123:585-595.

638 Tokarska, M., T. Marshall, R. Kowalczyk, J. M. Wójcik, C. Pertoldi, T. N. Kristensen, V. 639 Loeschcke, V. R. Gregersen, and C. Bendixen. 2009. Effectiveness of microsatellite and SNP 640 markers for parentage and identity analysis in species with low genetic diversity: the case of 641 European bison. Heredity 103:326-332. 
642 Väli, U., A. Einarsson, L. Waits, and H. Ellegren. 2008. To what extent do microsatellite

643 markers reflect genome-wide genetic diversity in natural populations? Mol. Ecol. 17:3808-3817.

644 Wang, J., E. Santiago, and A. Caballero. 2016. Prediction and estimation of effective population

645 size. Heredity 117:193-206.

646 Waples, R. K., W. A. Larson, and R. S. Waples. 2016. Estimating contemporary effective

647 population size in non-model species using linkage disequilibrium across thousands of loci.

648 Heredity 117:233-240.

649 Waples, R. S., T. Antao, and G. Luikart. 2014. Effects of overlapping generations on linkage 650 disequilibrium estimates of effective population size. Genetics 197:769-780.

651 Waples, R. S., and C. DO. 2008. ldne: a program for estimating effective population size from 652 data on linkage disequilibrium. Mol. Ecol. Resour. 8:753-756.

653 Willoughby, J. R., N. B. Fernandez, M. C. Lamb, J. A. Ivy, R. C. Lacy, and J. A. DeWoody. 654 2015. The impacts of inbreeding, drift and selection on genetic diversity in captive breeding 655 populations. Mol. Ecol. 24:98-110.

656 Willoughby, J. R., J. A. Ivy, R. C. Lacy, J. M. Doyle, and J. A. DeWoody. 2017. Inbreeding and 657 selection shape genomic diversity in captive populations: Implications for the conservation of 658 endangered species. PLoS ONE 12:e0175996.

659 Wisely, S. M., S. W. Buskirk, and M. A. Fleming. 2002. Genetic diversity and fitness in black660 footed ferrets before and during a bottleneck. Journal of ....

661 Witzenberger, K. A., and A. Hochkirch. 2011. Ex situ conservation genetics: a review of 662 molecular studies on the genetic consequences of captive breeding programmes for endangered 663 animal species. Biodivers. Conserv. 20:1843-1861.

664 Wright, D., C. J. Rubin, A. Martinez Barrio, K. Schütz, S. Kerje, H. Brändström, A. Kindmark, 665 P. Jensen, and L. Andersson. 2010. The genetic architecture of domestication in the chicken: 666 effects of pleiotropy and linkage. Mol. Ecol. 19:5140-5156.

667 Wright, S. 1948. On the roles of directed and random changes in gene frequency in the genetics 668 of populations. Evolution 2:279.

669 Wright, S. 1932. The roles of mutation, inbreeding, crossbreeding, and selection in evolution.

670 Zhdanova, O. L., and A. I. Pudovkin. 2008. Nb_HetEx: a program to estimate the effective

671 number of breeders. Journal of Heredity.

672

673 


\section{Figure 1 (on next page)}

A flowchart showing the known demographic history of the Richardsons Red Junglefowl

A graphical representation of the known population history of the Richardson's Red Junglefowl population in captivity and chain of custody. The red rimmed box denotes released populations that ultimately failed to take hold, and experienced extinction in their new habitat. The blue shaded boxes show the history of the populations that trace back to Isaac Richardson's flock. 
TIMELINE

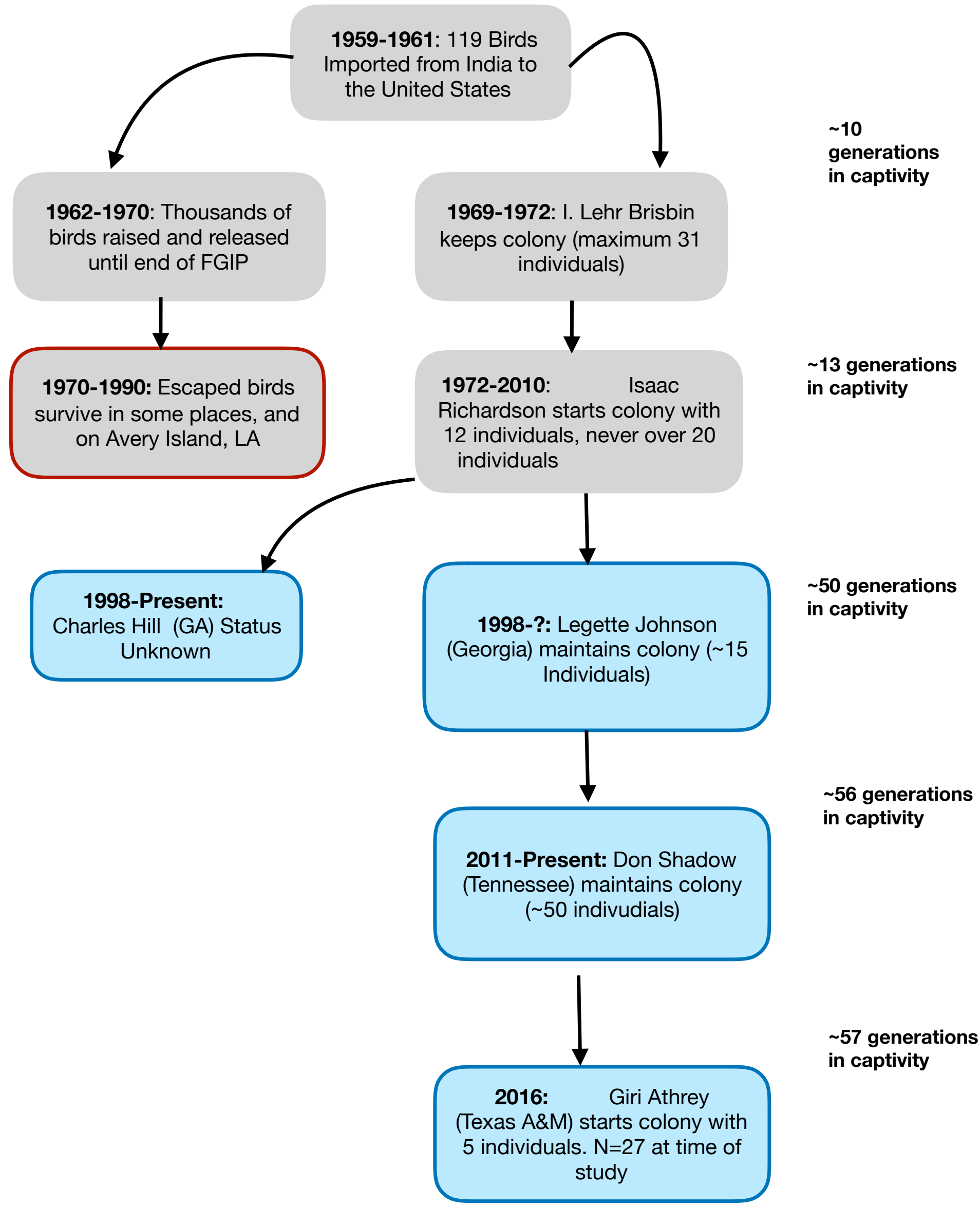




\section{Figure 2}

LD plot generated based on recombination frequencies across the MHC-B locus as characterized by the SNP panel.

A linkage map based on the 90 SNP loci on chromosome 16, generated from genotype data for the study population. The map shows pairwise estimates of linkage disequilibrium between markers. Red colored blocks suggest high linkage disequilibrium ( $\left.D^{\prime}\right)$ values of 1 , implying little to no recombination between markers.

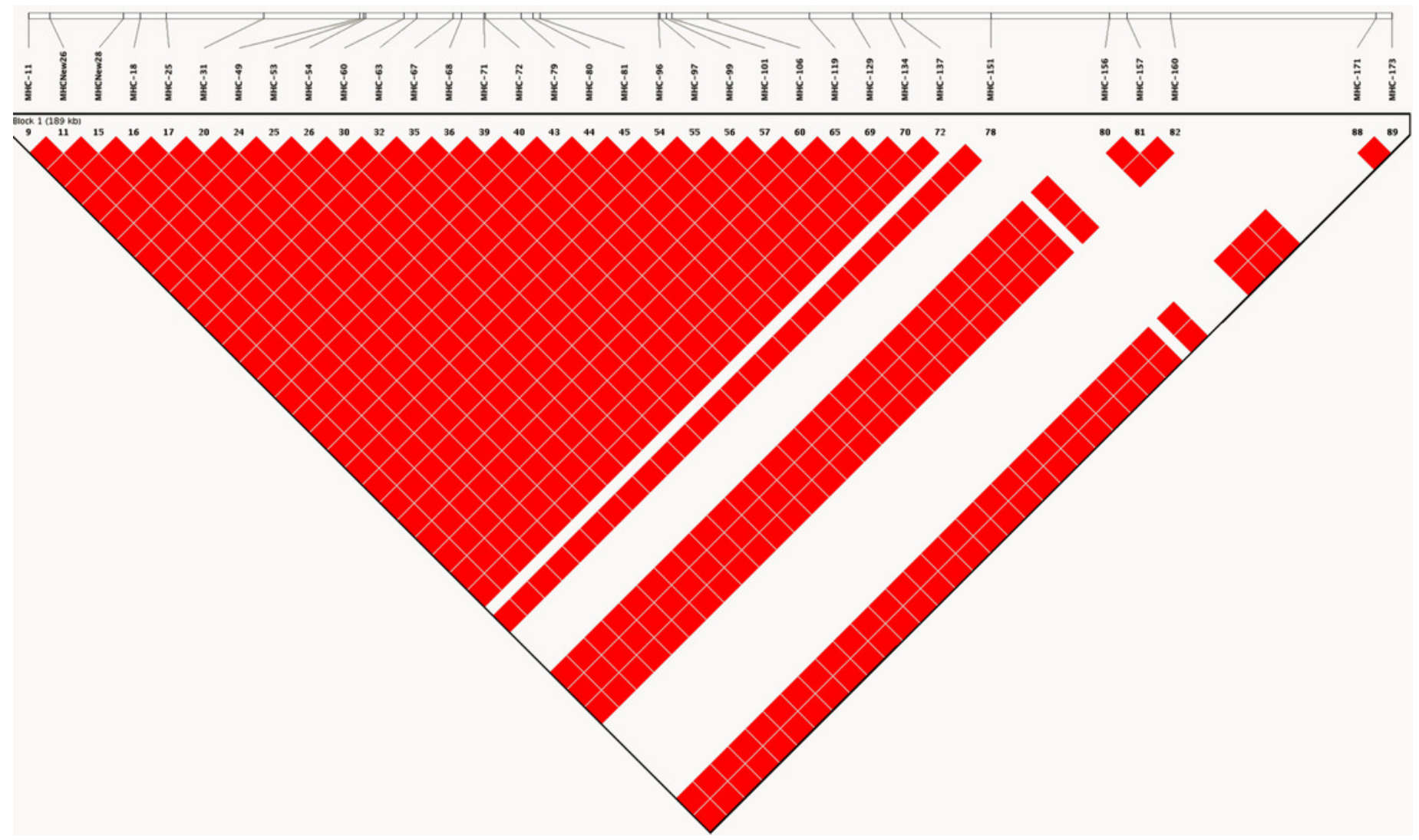


Figure 3 (on next page)

A haplotype network based on genetic distances between the five haplotypes identified in the study population.

A haplotype network of the the five (phased) haplotypes detected in the study population. Only three of the five unique haplotypes were found at a frequency of over $10 \%$ in the population (I, III, and V). 


\section{Table $\mathbf{1}$ (on next page)}

Summary of genetic diversity in the study population based on microsatellite and SNP loci.

Estimates of genetic diversity for both microsatellite and SNP datasets are presented. For each marker type, genetic diversity measures are shown along with their standard errors. The columns present the number of alleles ( $\mathrm{Na}$ ), effective number of alleles (Nae), observed heterozygosity (Ho), expected heterozygosity (He), the unbiased expected heterozygosity (uHe), and the fixation index $F$. 
1 Table 1: Estimates of genetic diversity for both microsatellite and SNP datasets are presented.

2 For each marker type, genetic diversity measures are shown along with their standard errors.

3 The columns present the number of alleles (Na), effective number of alleles (Nae), observed

4 heterozygosity (Ho), expected heterozygosity (He), the unbiased expected heterozygosity (uHe),

5 and the fixation index $F$.

$\begin{array}{cccccccc}\text { Marker Type } & & \text { Na } & \text { Nae } & \text { Ho } & \text { He } & \text { uHe } & \text { F } \\ \text { Microsatellite } & \text { Mean } & 2.33 & 1.74 & 0.36 & 0.37 & 0.38 & 0.07 \\ & \text { SE } & 0.16 & 0.14 & 0.06 & 0.05 & 0.05 & 0.08 \\ \text { SNP } & \text { Mean } & 1.38 & 1.13 & 0.11 & 0.1 & 0.1 & -0.14 \\ & \text { SE } & 0.05 & 0.02 & 0.01 & 0.01 & 0.01 & 0.02\end{array}$

6

7

8

9

10 


\section{Table 2 (on next page)}

Comparison of SNP based genetic diversity measures between study population, and other junglefowl and chicken studies.

Estimates of nucleotide diversity, haplotype number and diversity and Tajima's D in captive study population, compared against wild junglefowl populations and domestic chicken. All the studies compared here used the same high-density SNP panel covering the MHC-B locus on Chromosome 16. 
3 Table 2: Estimates of nucleotide diversity, haplotype number and diversity and Tajima's D in

4 captive study population, compared against wild junglefowl populations and domestic chicken.

5 All the studies compared here used the same high-density SNP panel covering the MHC-B locus

6 on Chromosome 16.

\begin{tabular}{|l|l|l|l|l|l|l|}
\hline Type & N & $\begin{array}{l}\text { Nucleotide } \\
\text { Diversity }\end{array}$ & $\begin{array}{l}\text { Haplotype } \\
\text { Diversity }\end{array}$ & $\begin{array}{l}\text { Unique } \\
\text { Haplotypes }\end{array}$ & $\begin{array}{l}\text { Tajima's } \\
\text { D }\end{array}$ & Source \\
\hline $\begin{array}{l}\text { Red } \\
\text { Junglefowl }\end{array}$ & 27 & 0.009 & $43 \%$ & 5 & -3.191 & This study \\
\hline $\begin{array}{l}\text { Red } \\
\text { Junglefowl } \\
\text { (5 wild } \\
\text { populations) }\end{array}$ & 199 & $0.28(\mathrm{avg})$ & $99 \%$ (avg) & 310 & 2.1 & $\begin{array}{l}\text { Nguyen-Phuc } \\
\text { et al (2016) }\end{array}$ \\
\hline $\begin{array}{l}\text { Finnish } \\
\text { Landrace } \\
\text { Chicken } \\
(12 \\
\text { populations) }\end{array}$ & 195 & Not available & $\begin{array}{l}\text { Not } \\
\text { Available }\end{array}$ & 36 & $\begin{array}{l}\text { Not } \\
\text { Available }\end{array}$ & $\begin{array}{l}\text { Fulton } \text { et al } \\
\text { (2017) }\end{array}$ \\
\hline $\begin{array}{l}\text { Domestic } \\
\text { chicken }(17 \\
\text { breeds) }\end{array}$ & 1351 & Not available & $\begin{array}{l}\text { Not } \\
\text { available }\end{array}$ & 86 & $\begin{array}{l}\text { not } \\
\text { available }\end{array}$ & $\begin{array}{l}\text { Fulton } \text { et al } \\
\text { (2016a) }\end{array}$ \\
\hline $\begin{array}{l}\text { Domestic } \\
\text { chicken } \\
\text { (5 breeds) }\end{array}$ & 112 & 0.05 & $74 \%$ & 31 & -2.12 & $\begin{array}{l}\text { Athrey et al } \\
\text { unpublished }\end{array}$ \\
\hline
\end{tabular}




\section{Table 3(on next page)}

Effective population size estimates for microsatellite and SNP markers in the study population

Estimates of effective population size Ne are presented for microsatellite and SNP datasets. Ne estimates from three estimators are shown along with their respective $95 \%$ confidence intervals. Ne was estimated using the Heterozygote Excess (NeHE), Linkage Disequilibrium (NeLD), and the Molecular Coancestry (NeMC) methods. 
1 Table 3: Estimates of effective population size Ne are presented for microsatellite and SNP

2 datasets. Ne estimates from three estimators are shown along with their respective 95\%

3 confidence intervals. Ne was estimated using the Heterozygote Excess (NeHE), Linkage

4 Disequilibrium (NeLD), and the Molecular Coancestry (NeMC) methods.

$\begin{array}{ccccccc} & \text { NeHE } & 95 \% \mathrm{CI} & \mathrm{NeLD} & 95 \% \mathrm{CI} & \mathrm{NeMC} & 95 \% \mathrm{CI} \\ \text { Microsatellite } & \text { inf } & 7.3 \text {-inf } & 7.3 & 3.6-12.5 & 4 & 1.1-8.8 \\ \text { SNP } & 4.8 & 3.1-11 & 0.2 & 0.1-0.2 & 1 & 1-1\end{array}$

